\title{
16 Ceramic Technology and Theoretical Perspectives
}

It should be clear by now that the study of pottery requires a precise and accurate characterisation of the pastes and fabrics in order to both identify the materials and techniques used in its production, and to interpret these technical actions within a society. In this sense, there are different theoretical approaches concerning the way technology is understood and how technological actions are interpreted that have triggered a marked dichotomy in the explanations about ceramic technology. In agreement with some of the major theoretical positions that have characterised archaeology since the second half of the twentieth century, some researchers have resorted to technical, environmental and functional reasons, while others have emphasised cultural, ideological and social aspects (Livingstone-Smith, 2000).

However, this theoretical divergence can be counterproductive for properly understanding the role of pottery along its life cycle. In this sense, social, symbolic and ideological aspects inherent in ceramics may interact and appear along with other practical and technical issues related to the materiality and natural environment. Thus, far from being eclectic, we have tried to take a position open to dialogue and away from dogmas when dealing with the main theoretical trends that are currently applied in the interpretation of pottery. The aim is to reflect on their possibilities and limitations, highlighting the most interesting contributions that can be derived from them.

As pointed out by Vidal et al. (2011), the complexity of facing the intangible reality underlying the objects of past societies makes it necessary to apply a wide range of criteria and approaches in the study of pottery. In this sense, neither methodological and theoretical frameworks nor spatial or temporal backgrounds should be neglected. In the current fragmentation observed in ceramic studies, all perspectives have some constraints but also certain positive points that, once considered, provide more complex insights regarding the role of ceramics in society. Therefore, it may be unwise to adopt a dogmatic theoretical position, since we start from the idea that the relationships existing between pottery and people, as well as with other objects, are probably quite complex. Even accepting our own limitations as individuals to address this complexity, we have to be aware that the use of different theoretical perspectives might also lead to issues that can be often closely correlated. Each theoretical trend provides specific information and a particular viewpoint about the many dimensions of pottery, but it is only through the combination of several theoretical and methodological approaches that we can approach all the issues interrelated as a whole (García Rosselló, 2008; Van der Leeuw, 1999).

This chapter focuses on the three main theories currently applied in the interpretation of ceramic technology through data obtained from the archaeometric analysis of pottery fabrics: ceramic ecology, functionalism and the social theory of technology. Some of these theoretical perspectives have common interests while others are epistemologically divergent. In this sense, many of the topics addressed 
by one or another position coincide, such as the shared interest in approaching raw material procurement, although they differ in the viewpoint used and the relevance given to the different aspects studied. The first two theoretical approaches can be generically classified within evolutionary, normative, materialistic, essentialist and deterministic trends based on a rationalist logic much closer to the natural sciences. In these discourses an interest in establishing testable, reproducible and objectively established cross-cultural general laws usually exists.

There is an extensive literature (e.g., Arnold, 1985; Braun, 1983; Hoard et al., 1995; O’Brien et al., 1994; Rye, 1976; Schiffer, 1990b; Schiffer and Skibo, 1987) in which both evolutionary perspectives, ceramic ecology and functionalism, are combined. However, some differences regarding the issues in which they focus, along with certain interpretive nuances (O'Brien et al., 1998), allow us to divide this broad evolutionary trend into two distinct positions. As pointed by P. Rice (1996), there are differences regarding the interpretation of technological features. On the one hand, a technological trait can be related to economic viewpoints which determine the pottery production and are typical of ceramic ecology. On the other, the same technological trait can be interpreted according to high efficiency assumptions characteristic of functionalist positions more related to pottery use. Although we must be aware that both perspectives often go hand in hand, the different approaches stated within the general trend suggest treating both tendencies separately.

Opposed to these perspectives, we will also focus on another viewpoint on technology with an origin linked to the school of Techniques et Culture and the anthropology of techniques. For the last two decades the conceptual foundations of these lines of thought have been also combined with propositions from postprocessual (contextual) archaeology. In this sense, it is a point of view on technology that is closer to the social sciences and owns a relativistic background regarding the way knowledge is generated, thus considering the multivocality and incommensurability involved in the different ways of seeing the world (Jones, 2002). It is a perspective in which there is, therefore, a greater interest in intra-cultural aspects and in generating specific and deeply contextual discourses. 